\title{
Non-invasive scoring systems for predicting NASH in Japan: evidences from Japan Study Group of NAFLD
}

Yoshio Sumida $^{1 *}$, Atsushi Nakajima ${ }^{2}$, Hideyuki Hyogo ${ }^{3}$, Saiyu Tanaka ${ }^{4}$, Masafumi Ono ${ }^{5}$, Hideki Fujii ${ }^{6}$, Yuichiro Eguchi ${ }^{7}$, Masato Yoneda $^{8}$, Takeshi Okanoue ${ }^{9}$, and Yoshito Itoh ${ }^{1}$ and Japan Study Group of NAFLD (JSG-NAFLD)

${ }^{1}$ Department of Gastroenterology and Hepatology, Kyoto Prefectural University of Medicine, Kyoto, Japan

${ }^{2}$ Division of Gastroenterology, Yokohama City University Graduate School of Medicine, Yokohama, Japan

${ }^{3}$ Department of Medicine and Molecular Science, Graduate School of Biomedical Sciences, Hiroshima University, Hiroshima, Japan

${ }^{4}$ Center for Digestive and Liver Diseases, Nara City Hospital, Nara, Japan

${ }^{5}$ Department of Gastroenterology and Hepatology, Kochi Medical School, Kochi, Japan

${ }^{6}$ Department of Gastroenterology, Osaka City Juso Hospital, Osaka, Japan

${ }^{7}$ Department of Internal Medicine, Saga Medical School, Saga University, Saga, Japan

${ }^{8}$ Schiff Center for Liver Diseases, University of Miami Miller School of Medicine, FL, USA

${ }^{9}$ Hepatology Center, Saiseikai Suita Hospital, Osaka, Japan

\begin{abstract}
A disease spectrum of nonalcoholic fatty liver disease (NAFLD) ranges from nonalcoholic fatty liver (NAFL), a benign condition to nonalcoholic steatohepatitis (NASH) which can lead to liver-related mortality such as hepatocellular carcinoma, hepatic failure, and portal hypertension. Although liver biopsy is the gold standard for the diagnosis of NASH, it has several drawbacks such as complication, cost, and sampling errors. A reliable and inexpensive noninvasive parameter for predicting NASH or severe hepatic fibrosis is required in patients with NAFLD. In this article, we reviewed several scoring systems which have been constructed by the Japan Study Group of NAFLD (JSG-NAFLD), a multi-center study group for the diagnosis and management of NAFLD in Japan. First of all, the NAFIC score calculated from the ferritin, insulin, and type 4 collagen $7 \mathrm{~S}$ levels was established to diagnose NASH. We recently suggest that modified NAFIC score may be better than conventional NAFIC score, although external validation studies using a large population of NAFLD are essential. In order to exclude severe fibrosis (stage 3-4), FIB4 index and NAFLD fibrosis score (NFS) are the most simple and reliable scoring systems in consistent with the Western studies. The combination of platelet count and AST/ALT ratio (PAAR index) is useful and easily determined for excluding severe fibrosis. To detect cirrhosis (stage 4), we formed an easily calculated composite score, called the PLALA (platelet, albumin, AST/ALT ratio) score. Introduction of these scores in clinical practice may reduce the proportion of patients that require liver biopsy to diagnose mild disease.
\end{abstract}

\section{Introduction}

Nonalcoholic fatty liver disease (NAFLD) is an important cause of chronic liver injury in many countries including Japan. NAFLD represents a wide spectrum of conditions that are characterized histologically by macrovesicular hepatic steatosis ( $>5 \%$ of hepatocytes affected), and the diagnosis is made after excluding a history of consumption of alcohol in amounts sufficient to be considered harmful to the liver. NAFLD ranges over a wide spectrum, extending from nonalcoholic fatty liver (NAFL) which is generally benign, through to nonalcoholic steatohepatitis (NASH) to liver cirrhosis, end-stage liver disease, and even hepatocellular carcinoma (HCC) despite the absence of significant alcohol consumption [1]. The probability of developing advanced fibrosis and HCC is significantly greater in individuals with NASH than in those with NAFL. Liver biopsy as the gold standard tool of NASH can reveal the histologic steatosis with hepatocyte ballooning. However, it is difficult to perform liver biopsy for every patient with NAFLD to ascertain the presence of NASH and determine the stage and grade of the disease. In 2010, we established the Japan Study Group of NAFLD (JSG-NAFLD), represented by the following 10 hepatology centers: Yokohama City University, Asahikawa Medical College, Kurume University, Nara City Hospital, Hiroshima University, Saga Medical School, Osaka City University, Kyoto Prefectural University of Medicine, Kochi Medical School, and Saiseikai Suita Hospital. In 2014, JSG-NAFLD includes eleven hepatology centers through all over Japan (Figure 1). The aim of our study group is 1) to clarify prevalence, natural history, and prognosis in Japanese NAFLD, 2) to establish and validate parameters or scoring systems for the noninvasive diagnosis of NASH or severe fibrosis, and 3) to develop the pharmacological treatments for NASH.

Correspondence to: Yoshio Sumida, M.D., Ph.D, Department of Gastroentrology and Hepatology, Kyoto Prefectural University of Medicine, KawaramachiHirokoji, Kamigyo-ku, Kyoto 602-8566, Japan, Tel: +81-75-251-5519; Fax: +8175-251-0710; E-mail: sumida@koto.kpu-m.ac.jp

Key words: nonalcoholic steatohepatitis, hepatic fibrosis, platelet, ferritin; insulin, type 4 collagen $7 S$

Received: March 20, 2015; Accepted: April 01, 2015; Published: April 06, 2015 


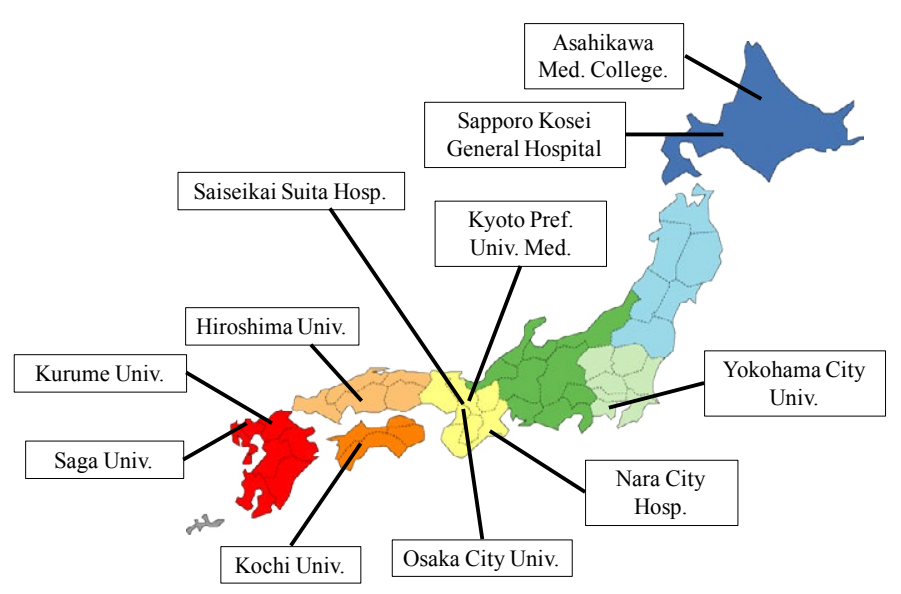

Figure 1. Japan Study Group of NAFLD (JSG-NAFLD).

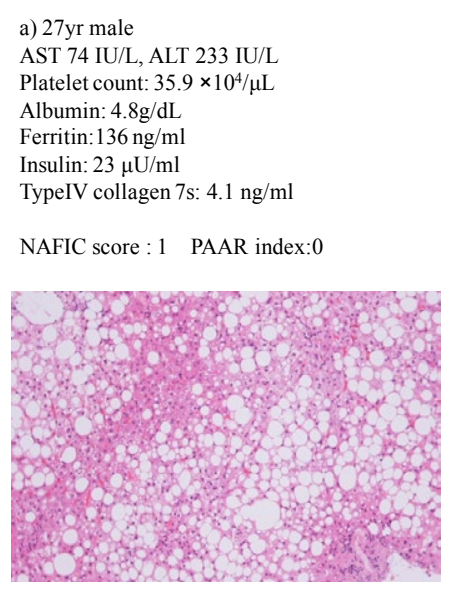

NAFL b) $57 \mathrm{yr}$ female

AST $38 \mathrm{IU} / \mathrm{L}$, ALT $40 \mathrm{IU} / \mathrm{L}$

Platelet count: $19.1 \times 10^{4} / \mu \mathrm{L}$

Albumin: $4.7 \mathrm{~g} / \mathrm{dL}$

Ferritin: $342 \mathrm{ng} / \mathrm{mL}$

Insulin: $14.3 \mu \mathrm{U} / \mathrm{mL}$

TypeIV collagen $7 \mathrm{~s}: 5.6 \mathrm{ng} / \mathrm{mL}$

NAFIC score: 4 PAAR index: 2

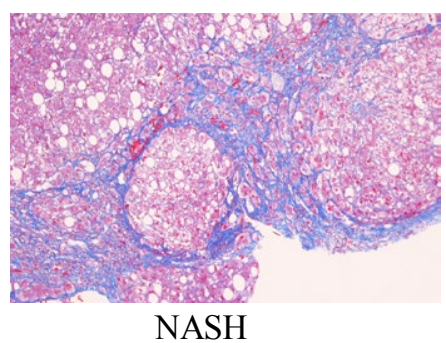

NASH
Figure 2. ALT does not help for predicting NASH.

\section{Scoring systems for predicting NASH in NAFLD}

Several lines of evidences have confirmed that NASH cannot be differentiated from NAFL by routine clinical parameters. Serum transaminase activities are known to be usually elevated in patients with NAFLD, but those levels do not always reflect severity of NAFLD. Two representative cases were shown in Figure 2. A 27 year-male patient with NAFLD had elevated transaminase activities (AST/ALT 74/233 IU/L). His histological examination revealed hepatic steatosis $(>66 \%$ of hepatocytes affected). He was diagnosed NAFL (Matteoni type 2, NAS score: $3+1+0=4$ ) (Figure $2 \mathrm{a}$ ). On the other hand, a 57 year-female patient with NAFLD almost mildly elevated levels of transaminase activities (AST/ALT 38/40 IU/L). Her liver histology revealed moderate steatosis with hepatocyte ballooning and extensive fibrosis. She was diagnosed NASH stage 3-4 (Matteoni type 4, NAS score: $2+1+2=5$ ) (Figure 2b). A variety of parameters has been suggested to detect NASH [2], including oxidative stress markers (thioredoxin, lipidperoxides), iron-related parameters (ferritin), hepatic fibrosis markers (type 4 collagen $7 \mathrm{~s}$, procollagen III peptide), and insulin resistance (HOMA-IR), adipokines (adiponectin, leptin), and apoptosis markers (soluble Fas, cytokeratin 18 (CK-18)). Among those, circulating levels of CK18 fragments have been investigated extensively as novel biomarkers for the presence of steatohepatitis in patients with
NAFLD [3]. Although these are very encouraging results, currently this assay is not commercially available. Furthermore, as each study utilized a study-specific cut-off value, there is not an established cutoff value for identifying steatohepatitis. According to the guidelines of the American Association for the Study of Liver Diseases (AASLD) [1], CK18 is not recommended in routine clinical practice. The first aim of JSG-NAFLD is to construct a simple index for detecting NASH among a large population of NAFLD in Japan. NAFIC score assigns one point for 200 (female) or 300 (male) ng/ml or higher ferritin, one point for 10 $\mu \mathrm{U} / \mathrm{ml}$ or higher fasting insulin, and two points for $5.0 \mathrm{ng} / \mathrm{ml}$ or higher type 4 collagen $7 \mathrm{~S}$. The total of these points is regarded as the NAFIC score (Table 1), and the possibility of NASH is high when the score is two or higher [4]. NAFIC score was 1 in the NAFL case (Figure 2a), while that was 4 in the NASH case (Figure 2b). A limitation of NAFIC score is low sensitivity and negative predictive value (NPV). At a cutoff value of NAFIC score 2, sensitivity, specificity, positive predictive value (PPV), and NPV were 60,87, 85, and 64\%, respectively. We reported the usefulness of the modified NAFIC scoring system, modified by changing the weightage assigned to the serum insulin levels, to obtain a reduced number of false-negative cases. Modified NAFIC is calculated as follows: serum ferritin $\geq 200 \mathrm{ng} / \mathrm{ml}$ (female) or $\geq 300 \mathrm{ng} / \mathrm{ml}$ (male), 1 point; serum type IV collagen $7 \mathrm{~s} \geq 5.0 \mathrm{ng} / \mathrm{ml}, 2$ points; serum fasting insulin $10-15 \mu \mathrm{U} / \mathrm{ml}, 1$ point and $\geq 15 \mu \mathrm{U} / \mathrm{ml}$, 2 points. The modified NAFIC score showed improved sensitivity and NPV for the diagnosis of NASH [5]. Three parameters including serum ferritin, insulin, type4 collagen $7 \mathrm{~S}$ can help us pick up NASH in NAFLD.

\section{Scoring systems for predicting severe fibrosis in NAFLD}

Since patients with severe fibrosis (stage 3 or 4 ) are at high risk for the development of HCC [6,7], noninvasive diagnosis of severe fibrosis is one of the fields that has evolved most rapidly in recent years [8]. The NAFLD fibrosis score (NFS) [9], BARD score [10], APRI (AST to platelet ratio index) [11], and FIB4 index [12] are among the more widely investigated non-invasive tools to cross-sectionally predict advanced fibrosis in NAFLD. NFS is comprised of six parameters such as diabetes/impaired fasting glucose, age, AST, ALT, platelets, BMI, and albumin (http://www.nafldscore.com/). Of the four scoring systems, NFS has received the most extensive validation $[4,13,14]$ and it has been recommended for clinical use in the recent practice guideline on the diagnosis and management of NAFLD [1]. The BARD score developed by Harrison et al. represents the weighted sum of three easily available variables ( $\mathrm{BMI} \geq 28 \mathrm{~kg} / \mathrm{m}^{2}$ [ $=1$ point], $\mathrm{AAR} \geq 0.8$ [ $=2$ points], and DM [ $=1$ point]), and the authors demonstrated that a score of 2-4 was associated with an odds ratio of 17 for predicting advanced fibrosis [10]. In Japan, however, Fujii and colleagues reported significantly poorer applicability of BARD in Japanese patients with NAFLD, compared with Caucasian subjects [15]. BARD score is less predictive of advanced fibrosis in Japanese NAFLD patients because they are less obese than those in western countries. Another disadvantage of BARD score is low PPV. The BARDI score, an enhanced model of BARD, was created by adding the international normalized ratio (INR) to the BARD score [16]. The BARDI score had an improved PPV over the BARD score and maintained an excellent NPV. APRI was calculated as the following formula: APRI=(AST/upper limit)/platelet count [17]. The area under receiver operating characteristic (ROC) curve for APRI was 0.85 with an optimal cut-off of 0.98 , giving a sensitivity of $75 \%$ and a specificity of $86 \%$. The usefulness of APRI is equal to that of NFS for predicting severe fibrosis in NAFLD [11]. In contrest, the diagnostic accuracy of APRI or BARD score seems to be inferior to other complex invasive scoring systems [18]. FIB4 index (http://gihep.com/ 
Table 1. Formula of noninvasive scoring systems for detecting NASH or severe fibrosis.

\begin{tabular}{|c|c|}
\hline Formula & Equation \\
\hline NAFIC score & $\begin{array}{l}\text { Ferritin } \geq 200(\text { female }) \text { or } 300(\text { male }) \mathrm{ng} / \mathrm{ml}=1 \text { point } \\
\text { Insulin } \geq 10 \mu \mathrm{U} / \mathrm{ml}=1 \text { point } \\
\text { Type IV collagen } 7 \mathrm{~s} \geq 5.0 \mathrm{ng} / \mathrm{ml}=2 \text { points } \\
\text { Scale: } 0-4\end{array}$ \\
\hline Modified NAFIC & $\begin{array}{l}\text { Ferritin } \geq 200(\text { female }) \text { or } 300(\text { male }) \mathrm{ng} / \mathrm{ml}=1 \text { point } \\
\text { Insulin } \geq 10 \mu \mathrm{U} / \mathrm{ml}=1 \text { point, } \geq 15 \mu \mathrm{U} / \mathrm{ml}=2 \text { points } \\
\text { Type IV collagen } 7 \mathrm{~s} \geq 5.0 \mathrm{ng} / \mathrm{ml}=2 \text { points } \\
\text { Scale: } 0-5\end{array}$ \\
\hline NAFLD fibrosis score & $\begin{array}{l}-1.675+0.037 \times \text { age }(\text { years })+0.094 \times \mathrm{BMI}\left(\mathrm{kg} / \mathrm{m}^{2}\right)+1.13 \times \mathrm{IFG} / \text { diabetes }(\mathrm{yes}=1, \text { no }=0)+0.99 \times \mathrm{AST} / \mathrm{ALT} \text { ratio }(\text { AAR })-0.013 \times \text { platelet } \\
\text { count }\left(\times 10^{\%} / \mathrm{L}\right)-0.66 \times \text { albumin }(\mathrm{g} / \mathrm{dL}) .\end{array}$ \\
\hline FIB4 index & $($ Age $[$ years $] \times$ AST $[\mathrm{IU} / \mathrm{L}]) /\left(\right.$ platelet count $\left.\left[\times 10^{9} / \mathrm{L}\right] \times \sqrt{\mathrm{ALT}}[\mathrm{IU} / \mathrm{L}]\right)$ \\
\hline PAAR index & $\begin{array}{l}\text { Platelet }<19.5 \times 10^{4} / \mu \mathrm{L}=1 \text { point } \\
\text { AST/ALT ratio }(\mathrm{AAR}) \geq 0.8=1 \text { point } \\
\text { Scale } 0 \text { - } 2\end{array}$ \\
\hline PLALA score & $\begin{array}{l}\text { Platelet }<15.3 \times 10^{4} / \mu \mathrm{L}=1 \text { point } \\
\text { Albumin }<4.0 \mathrm{~g} / \mathrm{dL}=1 \text { point } \\
\text { AST/ALT ratio }(\mathrm{AAR}) \geq 0.9=1 \text { point } \\
\text { Scale } 0-3\end{array}$ \\
\hline
\end{tabular}

calculators/hepatology/fibrosis-4-score/) is a biomarker panel using Age, AST, platelet count, and ALT (FIB4 index=age $(\mathrm{yr}) \times$ AST $(\mathrm{IU} / \mathrm{L}) /$ platelet count $(109 / \mathrm{L}) \times \sqrt{ }$ ALT $(\mathrm{IU} / \mathrm{L}))$, although derived in patients with HCV/HIV co-infection [19]. In four separate validation studies in NAFLD subjects in the United States $(\mathrm{n}=541)$, the United Kingdom $(\mathrm{n}=145)$, France and Hong Kong $(\mathrm{n}=246)$, and Japan $(\mathrm{n}=576)$, FIB4 index appeared to have the highest AUROC among all tested clinical prediction scores for detecting severe fibrosis [12,20-22]. FIB4 has been known to be one of the best parameters for predicting advanced fibrosis even in diabetic patients [23], children [24], and Latin [25] or Asian population [26]. However it is unknown whether these scoring systems are useful even in NAFLD patients showing normal ALT levels, because ALT level is sometimes within upper normal limit even in patients with severe fibrosis. FIB4 index has been observed to detect or exclude severe fibrosis even in NAFLD patients with normal ALT levels $[27,28]$.On the basis of these results, the guideline committee of Japan Society of Gastroenterology recommended the FIB4 index and NFS to detect severe fibrosis in Japanese patients with NAFLD. The distribution of FIB4 index was examined in 5,410 people with NAFLD who were diagnosed at health checkups. Overall, 4729 (87.4\%) had FIB4 index below the low cut-off point $(<1.45), 654(12.1 \%)$ had FIB4 index between indeterminate range (1.45-3.25) and $27(0.5 \%)$ had FIB4 index above the high cut-off point $(>3.25)[29,30]$. This result suggested about $90 \%$ of NAFLD patients were unlikely to have severe fibrosis. NFS and FIB4 index have several drawbacks. First, these scoring systems can be cumbersome and difficult to apply in every practice, although the relevant details can easily be entered onto a pre-designed Excel spreadsheet that can produce an instant result in front of the patient. Second, it is possible that the aged patients with NAFLD can be over-staged, since these indexes include age. With increasing hepatic fibrosis the ALT typically falls and the AST remains stable or rises, and as a result the AAR increases and can be a useful simple method of identifying patients with fibrosis. Platelet count has been known to decrease with increasing hepatic fibrosis in chronic liver disease. Previously we reported that the platelet count may be an ideal biomarker of the severity of fibrosis also in NAFLD patients on the basis of a multicenter study by the JSG of NAFLD [30]. Thus, we have hypothesized that the combination of platelet count and AAR can be reliable and simple parameters for predicting severe fibrosis in NAFLD. NAFLD patients with platelet count $>19.5 \times 10^{4} /$ $\mu \mathrm{L}$ and $\mathrm{AAR}<0.80$ (PAAR index $=0$ ) are unlikely to have advanced

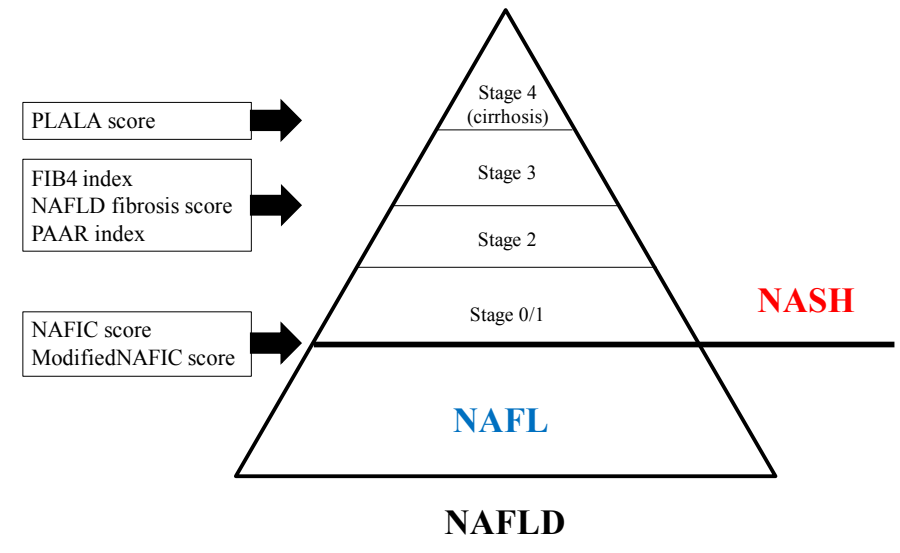

Figure 3. Non-invasive scoring systems to detect NASH or severe fibrosis.

fibrosis (NPV: $98 \%$ in the estimation group and $90 \%$ in the validation group). The combination of platelet and AAR, we call PAAR index, is useful and easily determined even in routine clinical practice or health checkups. PAAR index was 0 in the NAFL case (Figure 2a) and 2 in the NASH case (Figure 2b). The platelet count and AAR (PAAR index) in NAFLD patients seems to be an ideal biomarker of the severity of fibrosis, because it is simple, easy to measure and handle, cost-effective, and accurate for diagnosis of the severity of fibrosis.

\section{Scoring systems for predicting cirrhosis in NAFLD}

At first, Fujii and colleagues who are members of JSG-NAFLD, confirmed the clinical usefulness of two scoring systems such as cirrhosis discriminant score (CDS), and the hepatitis $\mathrm{C}$ antiviral long-term treatment against cirrhosis (HALT-C) model [31]. These scores consist of platelet count, AAR, and PT-INR. Our study group (JSG-NAFLD) tried to originally develop a simple scoring system to differentiate cirrhosis from non-cirrhosis in NAFLD patients. The three variables platelet, albumin, and AAR were combined in an unweighted sum (platelet $<15.3 \times 10^{4} / \mu \mathrm{L} ; 1$ point, serum albumin $<4.0$ $\mathrm{g} / \mathrm{dL} ; 1$ point, and AAR $>0.9 ; 1$ point) and formed an easily calculated composite score for predicting cirrhosis in NAFLD patients, called the PLALA score. A PLALA score (2 and 3) was useful for detecting liver cirrhosis in NAFLD patients (sensitivity, $86.8 \%$; specificity, $90.8 \%$; NPV, 99.5\%; PPV, 26.2\%) (Figure 3) [32]. 


\section{Scoring systems for predicting all-cause or liver related mortality and systemic complications in NAFLD}

A major limitation of these scoring systems is that they have largely investigated in cross-sectional studies and thus their utility in monitoring disease natural history, predicting outcomes or response to therapeutic intervention remains unknown. Ideal non-invasive scoring systems can predict all-cause or liver related mortality and systemic complications in NAFLD. It was recently reported that the scores derived from the fibrosis-predicting scoring systems NFS, APRI, and FIB4 index also serve as prognostic factors [33]; the prognostic values of these scores still need to be verified in Japan. In Japan, Kawamura et al. reported that the annual liver carcinogenic rate in NAFLD patients was $0.043 \%$, and that APRI was useful in predicting liver carcinogenesis [34].Among previously established scoring systems, NFS is the best predictor for mortality in US and China [35-37]. NFS and FIB4 index could identify patients at risk of onset of DM and cerebral-cardiovascular diseases [38]. In the future, we should clarify if our proposed scoring systems (NAFIC score, PAAR index, and PLALA score) predict cancer incidence (hepatic/extrahepatic) and all-cause or liver-related mortality.

\section{Conclusions}

In this article, we extensively reviewed several scoring systems to detect NASH or severe fibrosis in NAFLD, mainly on the basis of data from the JSG-NAFLD study in Japan. Although external validation or longitudinal studies are required for practical use, these scoring systems can be easily determined and useful for the management of NAFLD (Figure 3).

\section{References}

1. Chalasani N, Younossi Z, Lavine JE, Diehl AM, Brunt EM, et al. (2012) The diagnosis and management of non-alcoholic fatty liver disease: practice guideline by the American Gastroenterological Association, American Association for the Study of Liver Diseases, and American College of Gastroenterology. Gastroenterology 142:1592-1609.

2. Sumida Y, Eguchi Y, Ono M (2010) Current status and agenda in the diagnosis of nonalcoholic steatohepatitis in Japan. World J Hepatol 2: 374-383. [Crossref]

3. Feldstein AE, Wieckowska A, Lopez AR, Liu YC, Zein NN, et al. (2009) Cytokeratin-18 fragment levels as noninvasive biomarkers for nonalcoholic steatohepatitis: a multicenter validation study. Hepatology 50: 1072-1078. [Crossref]

4. Sumida Y, Yoneda M, Hyogo H, Yamaguchi K, Ono M, et al. (2011) A simple clinical scoring system using ferritin, fasting insulin, and type IV collagen $7 \mathrm{~S}$ for predicting steatohepatitis in nonalcoholic fatty liver disease. $J$ Gastroenterol 46: 257-268. [Crossref]

5. Nakamura A, Yoneda M, Sumida Y, Eguchi Y, Fujii H, et al. (2013) Modification of a simple clinical scoring system as a diagnostic screening tool for non-alcoholic steatohepatitis in Japanese patients with non-alcoholic fatty liver disease. J Diabetes Investig 4: 651-658. [Crossref]

6. Ekstedt M, Hagström H, Nasr P, Fredrikson M, Stål P, et al. (2014) Fibrosis stage is the strongest predictor for disease-specific mortality in NAFLD after up to 33 years of follow-up. Hepatology In press. [Crossref]

7. Seko Y, Sumida Y, Tanaka S, Taketani H, Kanemasa K, et al. (2014) Predictors of malignancies and overall mortality in Japanese patients with biopsy-proven nonalcoholic fatty liver disease. Hepatol Res. [Crossref]

8. Sumida Y, Nakajima A, Itoh Y (2014) Limitations of liver biopsy and non-invasive diagnostic tests for the diagnosis of nonalcoholic fatty liver disease/nonalcoholic steatohepatitis. World J Gastroenterol 20: 475-485. [Crossref]

9. Angulo P, Hui JM, Marchesini G, Bugianesi E, George J, et al. (2007) The NAFLD fibrosis score: a noninvasive system that identifies liver fibrosis in patients with NAFLD. Hepatology 45: 846-854. [Crossref]
10. Harrison SA, Oliver D, Arnold HL, Gogia S, Neuschwander-Tetri BA (2008) Development and validation of a simple NAFLD clinical scoring system for identifying patients without advanced disease. Gut 57: 1441-1447. [Crossref]

11. Kruger FC, Daniels CR, Kidd M, Swart G, Brundyn K, et al. (2011) APRI: a simple bedside marker for advanced fibrosis that can avoid liver biopsy in patients with NAFLD/NASH. S Afr Med J 101: 477-480. [Crossref]

12. Shah A, Lydecker A, Murray K, Tetri BN, Contos MJ, et al. (2009) Comparison of noninvasive markers of fibrosis in patients with nonalcoholic fatty liver disease. Clin Gastroenterol Hepatol 7: 1104-1112. [Crossref]

13. Musso G, Gambino R, Cassader M, Pagano G (2011) Meta-analysis: natural history of non-alcoholic fatty liver disease (NAFLD) and diagnostic accuracy of non-invasive tests for liver disease severity. Ann Med 43: 617-649. [Crossref]

14. Wong VW, Wong GL, Chim AM, Tse AM, Tsang SW, et al. (2008) Validation of the NAFLD fibrosis score in a Chinese population with low prevalence of advanced fibrosis. Am J Gastroenterol 103: 1682-1688. [Crossref]

15. Fujii H, Enomoto M, Fukushima W, Tamori A, Sakaguchi H, et al. (2009) Applicability of BARD score to Japanese patients with NAFLD. Gut 58: 1566-1567. [Crossref]

16. Lee TH, Han SH, Yang JD, Kim D, Ahmed M (013) Prediction of Advanced Fibrosis in Nonalcoholic Fatty Liver Disease: An Enhanced Model of BARD Score. Gut Liver 7: 323-328. [Crossref]

17. Wai CT, Greenson JK, Fontana RJ, Kalbfleisch JD, Marrero JA, et al. (2003) A simple noninvasive index can predict both significant fibrosis and cirrhosis in patients with chronic hepatitis C. Hepatology 38: 518-526. [Crossref]

18. Adams LA, George J, Bugianesi E, Rossi E, De Boer WB, et al. (2011) Complex noninvasive fibrosis models are more accurate than simple models in non-alcoholic fatty liver disease. J Gastroenterol Hepatol 26: 1536-1543.

19. Sterling RK, Lissen E, Clumeck N, Sola R, Correa MC, et al. (2006) Development of a simple noninvasive index to predict significant fibrosis in patients with $\mathrm{HIV} / \mathrm{HCV}$ co infection. Hepatology 43: 1317-1325. [Crossref]

20. Sumida Y, Yoneda M, Hyogo H, Itoh Y, Ono M, et al. (2012) Validation of the FIB4 index in a Japanese nonalcoholic fatty liver disease population. BMC Gastroenterol 12: 2. [Crossref]

21. McPherson S, Stewart SF, Henderson E, Burt AD, Day CP (2010) Simple non-invasive fibrosis scoring systems can reliably exclude advanced fibrosis in patients with nonalcoholic fatty liver disease. Gut 59: 1265-1269. [Crossref]

22. Wong VW, Vergniol J, Wong GL, Foucher J, Chan HL, et al. (2010) Diagnosis of fibrosis and cirrhosis using liver stiffness measurement in nonalcoholic fatty liver disease. Hepatology 51: 454-462. [Crossref]

23. Morling JR, Fallowfield JA, Guha IN, Nee LD, Glancy S, et al. (2014) Using noninvasive biomarkers to identify hepatic fibrosis in people with type 2 diabetes mellitus: the Edinburgh type 2 diabetes study. J Hepatol 60: 384-391. [Crossref]

24. Yang HR, Kim HR, Kim MJ, Ko JS, Seo JK (2012) Noninvasive parameters and hepatic fibrosis scores in children with nonalcoholic fatty liver disease. World J Gastroenterol 18: 1525-1530. [Crossref]

25. Pérez-Gutiérrez OZ, Hernández-Rocha C, Candia-Balboa RA, Arrese MA, Benítez C, et al. (2013) Validation study of systems for noninvasive diagnosis of fibrosis in nonalcoholic fatty liver disease in Latin population. Ann Hepatol 12: 416-424. [Crossref]

26. Xun YH, Fan JG, Zang GQ, Liu H, Jiang YM, et al. (2012) Suboptimal performance of simple noninvasive tests for advanced fibrosis in Chinese patients with nonalcoholic fatty liver disease. J Dig Dis 13: 588-95. [Crossref]

27. Yoneda M, Imajo K, Eguchi Y, Fujii H, Sumida Y, et al. (2013) Noninvasive scoring systems in patients with nonalcoholic fatty liver disease with normal alanine aminotransferase levels. $J$ Gastroenterol 48: 1051-1060. [Crossref]

28. McPherson S, Anstee QM, Henderson E, Day CP, Burt AD (2013) Are simple noninvasive scoring systems for fibrosis reliable in patients with NAFLD and normal ALT levels? Eur J Gastroenterol Hepatol 25: 652-658. [Crossref]

29. Sumida Y, Sakuragi S, Hibino S, Furutani M (2015) Distribution of the FIB4 index in Japanese nonalcoholic fatty liver disease population: a multi-center study. Ningen Dock International 2: 90-92.

30. Yoneda M, Fujii H, Sumida Y, Hyogo H, Itoh Y, et al. (2011) Platelet count for predicting fibrosis in nonalcoholic fatty liver disease. J Gastroenterol 46: 1300-1306. [Crossref] 
31. Fujii H, Enomoto M, Fukushima W, Ohfuji S, Mori M, et al. (2009) Noninvasive laboratory tests proposed for predicting cirrhosis in patients with chronic hepatitis $\mathrm{C}$ are also useful in patients with non-alcoholic steatohepatitis. $J$ Gastroenterol 44: 608614. [Crossref]

32. Kessoku T, Ogawa Y, Yoneda M, Imajo K, Sumida Y, et al. (2014) Simple scoring system for predicting cirrhosis in nonalcoholic fatty liver disease. World J Gastroentero 20: 10108-10114. [Crossref]

33. Kim D, Kim WR, Kim HJ, Therneau TM (2013) Association between noninvasive fibrosis markers and mortality among adults with nonalcoholic fatty liver disease in the United States. Hepatology 57: 1357-1365. [Crossref]

34. Kawamura Y, Arase Y, Ikeda K, Seko Y, Imai N, et al. (2012) Large-Scale long-term follow-up study of Japanese patients with non-alcoholic fatty liver disease for the onset of hepatocellular carcinoma. Am J Gastroenterol 107: 253-261. [Crossref]
35. Xun YH, Guo JC, Lou GQ, Jiang YM, Zhuang ZJ, et al. (2014) Non-alcoholic fatty liver disease (NAFLD) fibrosis score predicts 6.6-year overall mortality of Chinese patients with NAFLD. Clin Exp Pharmacol Physiol 41: 643-649. [Crossref]

36. Angulo P, Bugianesi E, Bjornsson ES, Charatcharoenwitthaya P, Mills PR, et al. (2013) Simple noninvasive systems predict long-term outcomes of patients with nonalcoholic fatty liver disease. Gastroenterology 145: 782-789. [Crossref]

37. Treeprasertsuk S, Björnsson E, Enders F, Suwanwalaikorn S, Lindor KD (2013) NAFLD fibrosis score: a prognostic predictor for mortality and liver complications among NAFLD patients. World J Gastroenterol 19: 1219-1229. [Crossref]

38. Takahashi Y, Kurosaki M, Tamaki N, Yasui Y, Hosokawa T, et al. (2014) Nonalcoholic fatty liver disease fibrosis score and FIB-4 scoring system could identify patients at risk of systemic complications. Hepatol Res. [Crossref]

Copyright: $\odot 2015$ Sumida Y. This is an open-access article distributed under the terms of the Creative Commons Attribution License, which permits unrestricted use, distribution, and reproduction in any medium, provided the original author and source are credited. 